\title{
Bloch-Maxwell treatment of amplification of high harmonic seed in soft x-ray laser amplifiers in both direct and chirped amplifications
}

\author{
Philippe Zeitoun ${ }^{\mathrm{a}, *}$, Eduardo Oliva ${ }^{\mathrm{a}, \mathrm{b}}$, Marta Fajardo ${ }^{\mathrm{a}, \mathrm{c}}$, David Ros ${ }^{\mathrm{d}}$, Stéphane Sebban ${ }^{\mathrm{a}}$, Pedro Velarde ${ }^{\mathrm{a}, \mathrm{b}}$ \\ a Laboratoire d'Optique Appliquée, ENSTA ParisTech-CNRS-Ecole Polytechnique ParisTech, Chemin de la hunière, 91671 Palaiseau, France \\ ${ }^{\mathrm{b}}$ Instituto de Fusion Nuclear, Universidad Politecnica de Madrid, Madrid, Spain \\ ${ }^{c}$ Centro de Fisica dos Plasmas, Instituto Superior Tecnico, Lisbon, Portugal \\ ${ }^{d}$ CLUPS, EA4127, Bat 106, Université Paris-Sud, 91405 Orsay, France
}

Keywords:

Soft x-ray laser

Chirped pulse amplification

High-order harmonic seeding

\begin{abstract}
A B S T R A C T
Seeding plasma-based soft x-ray laser (SXRL) demonstrated diffraction-limited, fully coherent in space and in time beam but with energy not exceeding $1 \mu \mathrm{J}$ per pulse. Quasi-steady-state (QSS) plasmas demonstrated to be able to store high amount of energy and then amplify incoherent SXRL up to several $\mathrm{mJ}$. Using 1D time-dependant Bloch-Maxwell model including amplification of noise, we demonstrated that femtosecond HHG cannot be efficiently amplified in QSS plasmas. However, using Chirped Pulse Amplification concept on HHG seed allows to extract most of the stored energy, reaching up to $5 \mathrm{~mJ}$ in fully coherent pulses that can be compressed down to $130 \mathrm{fs}$.
\end{abstract}

\section{Introduction}

The competition to obtain ultra-high intensities with infrared and visible lasers has moved to soft $(1-60 \mathrm{~nm})$ and hard $(<1 \mathrm{~nm})$ $\mathrm{x}$-ray wavelengths in the last decade. FLASH in Germany and recently LCLS in the USA have transformed X-ray science with the achievement of outstanding intensities between $10^{16}$ [1] and $10^{18} \mathrm{~W} \mathrm{~cm}^{-2}$ [2]. In the last few years, thanks to the unique combination of extremely high photon number per pulse $\left(\sim 10^{12}\right)$ and short pulse duration ( $<50 \mathrm{fs}$ ), new imaging techniques revealing structures and dynamics of matter at unprecedented time and space scales, the creation of new states of matter [3], non-linear $\mathrm{X}$-ray effects [4] have been demonstrated.

In the mid-80's and early 90's the "X-race" was dominated by laser-driven soft X-ray laser (SXRL) with the first evidence of strong lasing at $20.6 \mathrm{~nm}$ quickly followed by the first saturated SXRL. At this time, laser-driven soft $\mathrm{x}$-ray lasers held numerous promises for Biology, Chemistry, Solid-sate Physics, and Plasma physics [5]. Two decades later the panorama for laser-driven SXRLs is paradoxical. Major experimental advances have resulted in achievement of tabletop, picosecond soft x-ray lasers. Following an alternative route, the highest energy per pulse with $12 \mathrm{~mJ}[6]$ generated by laser-like soft X-ray sources is found at kJ class laser facilities. As a comparison the soft $\mathrm{x}$-ray free-electron laser FLASH routinely obtains energies of $\sim 20 \mu \mathrm{J} /$ pulse with maximum energies of $100 \mu \mathrm{J} /$ pulse. However, in comparison with FLASH, intensities achieved with laser-driven high-energy SXRL's remained surprisingly low at about $10^{13} \mathrm{~W} \mathrm{~cm}^{-2}$ [7] while one might expect to achieve intensities above $10^{18} \mathrm{~W} \mathrm{~cm}^{-2}$.

Thanks to record high energies per pulse, laser-driven soft x-ray lasers still hold outstanding promise, that will be realized when several critical issues are resolved. Multi-mJ SXRLs are running in the so-called amplification of spontaneous emission (ASE) mode, that is amplification of stochastic noise. To date ASE soft $\mathrm{x}$-ray lasers demonstrated only weak coherence. This can be improved by seeding the gain medium with a fully coherent external source, i.e., using high harmonic generation, in an SXRL amplifier overcame this bottleneck and demonstrated highly coherent soft X-ray lasers [8]. However, these experiments have been performed in plasmas unable to store high amounts of energy, and the result is the extraction of a maximum of $1 \mu \mathrm{J}$ per pulse [8,9]. To our knowledge, only one attempt has been made to seed a high-energy plasma, which resulted in but in complicated results [10], as the ASE fully dominated the output energy. Thus, the seeding of high energy, i.e., multi-millijoule, soft x-ray lasers was deemed impossible and abandoned. Here we propose to numerically explore the combination of high harmonic seeding and the so-called Chirped Pulse Amplification technique, transposed from the visible to the soft $\mathrm{x}$-ray range, as the unique to-date solution to generate a multimillijoule, femtosecond, fully coherent, diffraction-limited soft 
$\mathrm{x}$-ray laser. We will describe in detail the model of amplification of HHG seed and then explore the seeding of plasmas having highenergy storage in both direct and chirped HHG configurations.

\section{Simulations}

Seeding external ultra-fast ( $20 \mathrm{fs}$ ) soft $\mathrm{x}$-ray sources has the potential to achieve femtosecond laser-driven soft $\mathrm{x}$-ray lasers. However, up to now due to the low density and temperature of plasma amplifiers, the spectral width was too narrow, enlarging the seed duration to picosecond range after amplification. Recent numerical models demonstrated that seeding high density plasmas created by the interaction of intense laser with solid targets may generate soft $\mathrm{x}$-ray pulses with pulse lengths from 70 fs to $200 \mathrm{fs}$ [11]. These works pave a clear path to the production of ultra-short SXRLs, i.e., with large spectral width. Plasma amplifiers must simultaneously have ion temperatures in excess of $100 \mathrm{eV}$ and electron densities higher than several $10^{20} \mathrm{~cm}^{-3}$. Such conditions exist mainly in plasmas created by 100 s of ps to ns duration infrared or visible laser pulse. These plasmas are characteristic of the socalled "quasi-steady-state" (QSS) regime. Interestingly, the first seeding experiment was performed with such a plasma; but, with the surprising result that the amplification was weak ( 2 times) and the observed ASE dominated the amplified seed that was recorded on time-resolved detector.

Modelling of HHG seeding in QSS plasma has to be performed with a multi-scale treatment. Indeed, an exact description has to deal with three different characteristic timescales: $10-100 \mathrm{fs}$ for the amplified seed beam, $0.1-10 \mathrm{ps}$ for the atomic processes and $10-100 \mathrm{ps}$ for the envelop of the gain evolution. Consequently, this modelling requires the use of a time-dependent model explicitly coupling the different key levels of the lasing ion with the electromagnetic field of the amplified seed. Such model requires solving the time-dependent equations Bloch (population)-Maxwell (electromagnetic field), see Eqs. (1)-(4). Considering Ditmire and collaborators experiment [10], we note the very strong contribution of the amplified spontaneous emission. Consequently, our model explicitly includes the ASE as a second source, where the first is the seed.

We solved the one-dimensional time-dependent Maxwell-Bloch equations to describe the amplification of HHG seed beam through the plasma. Working with reduced time $(\tau=t-y / c)$ reduces these equations to a set of Ordinary Differential Equations, Eqs. (1)-(4). Polarization, induced by the electric field, depends on the dynamics of the level populations involved in the transition, on a depolarization rate proportional to collisions and a stochastic source term, which takes into account the spontaneous emission (Eq. (1)). This polarization amplifies the electric field when a population inversion is created. The populations involved in the transition have been computed using a three-level atomic system, taking into account the remaining atomic levels as a source or sink of population. The temporal behaviour of the collisional pumping is artificially adjusted so as to reproduce the Ditmire et al experimental data [10]. As a first step we observed that best adjustment is obtained by multiplying the collisional rates by a Gaussian profile with 200 ps FWHM. Full hydrodynamical modelling is under progress that will provide detailed spatiotemporal electron density and temperature, ion temperature as well as fractional ionization balance used as initial conditions of our Maxwell-Bloch code. The temporal resolution of level populations, combined with the treatment of the ASE allows for the study of several processes indispensable to understanding the amplification of HHG in a plasma, such as Rabi oscillations and amplification of a noisy signal (ASE) mixed with the harmonic signal. $\frac{\partial P}{\partial t}=-\gamma_{\mathrm{p}} P-i \frac{d^{2}}{\hbar} E\left(N_{2}-N_{1}\right)+\Gamma$

$$
\begin{aligned}
& \frac{\partial N_{2}}{\partial t}=-\sum_{x \neq 2} C_{2 x} N_{2}+C_{12} N_{1}+C_{02} N_{0}+\frac{1}{2 \hbar} \operatorname{Im}\left(E^{*} \cdot P\right) \\
& \frac{\partial N_{1}}{\partial t}=-\sum_{x \neq 1} C_{1 x} N_{1}+C_{21} N_{2}+C_{01} N_{0}-\frac{1}{2 \hbar} \operatorname{Im}\left(E^{*} \cdot P\right) \\
& \frac{\partial E}{\partial y}=\frac{i \omega_{0}}{2 c}\left(\frac{P}{\varepsilon_{0}}-\frac{\omega_{p}^{2}}{\omega_{0}^{2}} E\right)
\end{aligned}
$$

\section{Modelling femtosecond pumping of a high-energy plasma}

First, we try to reproduce numerically the Ditmire et al. [10] experiment to better understand the mechanisms behind the small amplification of the seed and low seed/ASE energy ratio. We believe these issues are critical for optimizing seeding experiment in QSS plasmas. As described in Ref. [10] the fraction of the seed beam energy effectively coupled with the gain region is rather low (0.1-0.5 nJ) due to mismatch between the seed and the lasing line spectral widths and the seed beam size being much larger than the gain region. We set the ion and electron temperature to $550 \mathrm{eV}$ and the electron density to $4 \times 10^{20} \mathrm{~cm}^{-3}$, as dictated by the Ditmire data [10]. Due to lack of accurate atomic data for Gallium, each calculation was performed on the $2 \mathrm{p}^{5} 3 \mathrm{~s} J=1 \rightarrow 2 \mathrm{p}^{5} 3 \mathrm{p} J=0$ line at $21.2 \mathrm{~nm}$ of neon-like zinc. These elements have similar atomic physic and hydrodynamic behaviours due to their close atomic weights ( $\mathrm{Ga}, Z=31$ and $\mathrm{Zn}, Z=30$ ). Figs. 1 and 2 show the temporal variation of total laser intensity (seed and ASE) and gain, respectively, versus the position along the amplifier. At the amplifier exit, the intensity reproduces the Ditmire experimental data. The seed is about 2 times stronger than the ASE, while the temporally integrated energy over the full emission time of the ASE reaches $5 \mathrm{~mJ}$, which dominates the seed energy of $11 \mu \mathrm{J}$.

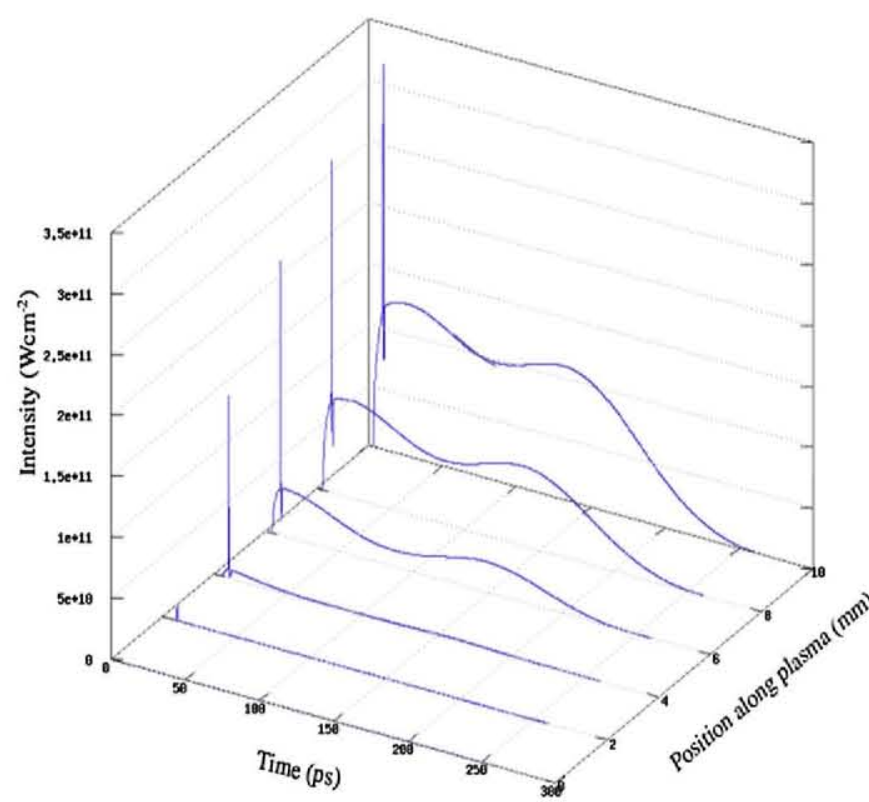

Fig. 1. Temporal evolution of the intensity for different planes along the amplifier. The spike is the harmonic slightly amplified while the long pulse is the ASE. 


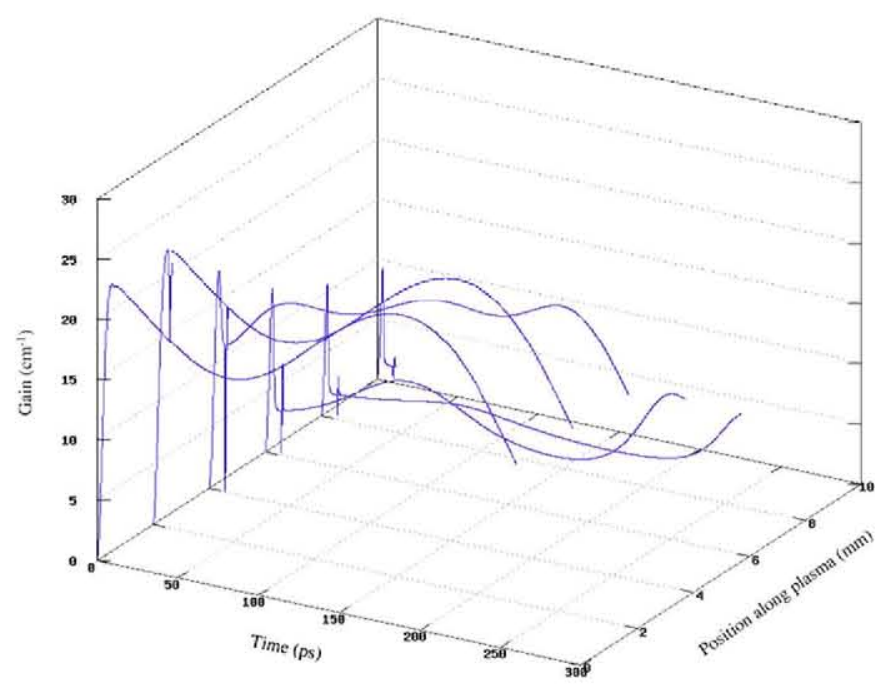

Fig. 2. Temporal evolution of the gain for different planes along the plasma. One may observe the gain saturation at about 10 ps due to the amplification of the HHG seed.

Two issues stand out in the gain spatio-temporal evolution. The gain is affected by the seed pulse amplification only over a very short duration of about $2 \mathrm{ps}$. This duration is longer than the seed pulse width, which is $20 \mathrm{fs}$ at the entrance and increases to $200 \mathrm{fs}$ at the exit, but much shorter than the $\sim 250$ ps gain duration itself. This means that $99 \%$ of the stored energy is not transferred to the soft $x$-ray laser and remains present in the plasma for amplification of the spontaneous emission after the 2 ps of seeded amplification. Also, we observed that the seed pulse saturates the gain at $4 \mathrm{~mm}$. For longer plasmas the ASE emitted before the injection of the seed deeply saturates the gain thus dramatically reducing the amplification of the seed. To fully clarify the respective role of ASE and seed, we modelled an optimized "Ditmire-like" experiment where the seed energy is 100 times $(0.1 \mu \mathrm{J})$ higher than that in the Ditmire experiment while keeping every other parameter unchanged. This can be easily achieved experimentally by focusing the seed beam and because of the progress recently achieved in high harmonic generation. The spatiotemporal variations of both the intensity and gain are displayed in Figs. 3 and 4, respectively. The differences

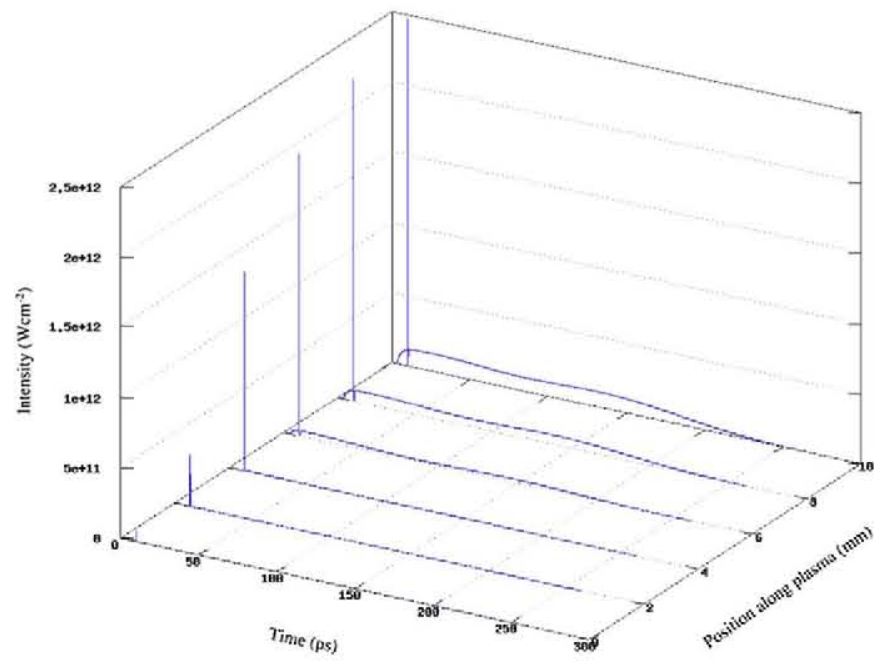

Fig. 3. Temporal evolution of intensity for different planes along the amplifier. The conditions are all similar to Fig. 1 but with an input energy increase by 100 times. The amplified HHG intensity dominates the output signal. However, after time integration, the ASE energy remains much stronger than the seed.

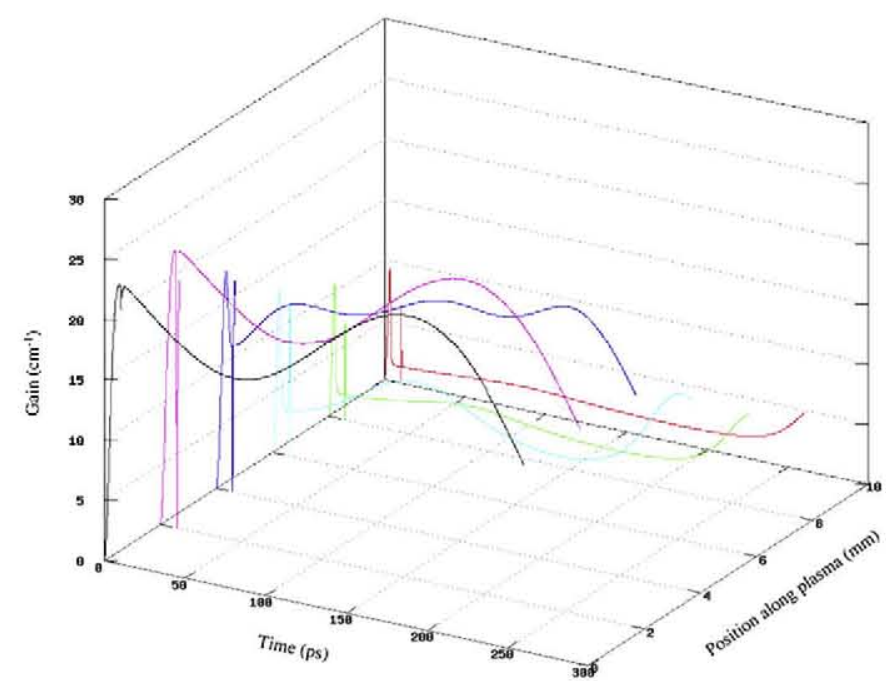

Fig. 4. Temporal evolution of the gain for different planes along the plasma amplifier.

with the previous case are striking. The seed pulse reaches an intensity of $2.5 \times 10^{12} \mathrm{~W} \mathrm{~cm}^{-2}$, i.e., 8 times higher than the $3.2 \times 10^{11} \mathrm{~W} \mathrm{~cm}^{-2}$ measured in the Ditmire experiment. However, the energy balance is not significantly improved with $17 \mu \mathrm{J}$ of amplified seed and $5 \mathrm{~mJ}$ on ASE. This result is easily explained considering the gain evolution along the plasma column. Again, for a long plasma ASE emitted before the seed saturates the gain, preventing amplification of the seed. In conclusion, we observe that even when seeding with the right conditions, i.e., with a seed intensity much higher than ASE, 99\% of the stored energy is not used for coherent seeding of the soft $\mathrm{x}$-ray laser and the ASE will dominate the time-integrated signal. Thus, it seems unproductive to seed QSS plasmas with femtosecond pulses.

\section{Modelling femtosecond pumping of a high-energy plasma}

The path for overcoming this bottleneck now appears straightforward: the seed beam should have the same duration as the gain and its intensity upon entrance to the plasma should be higher than

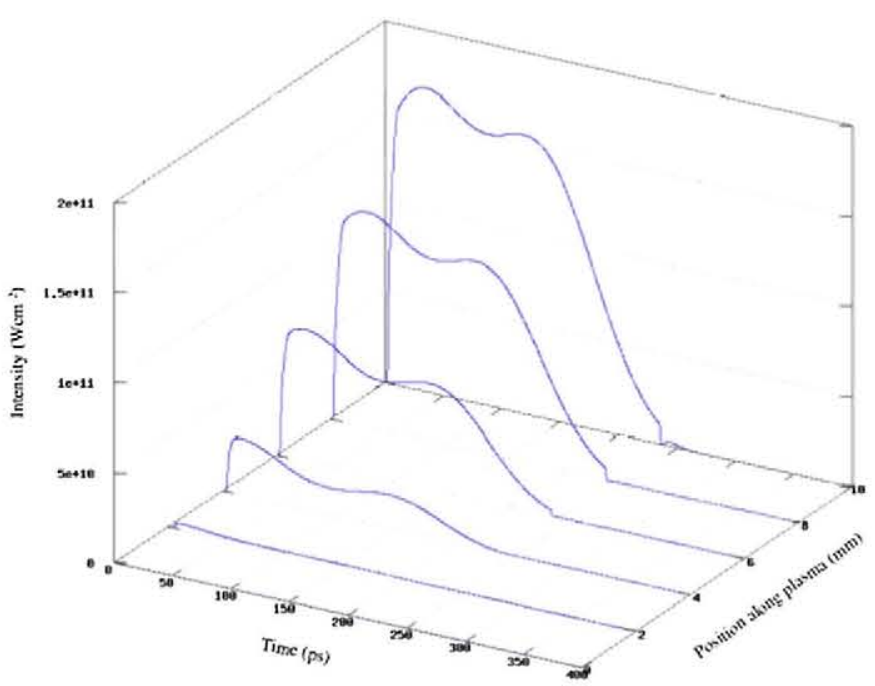

Fig. 5. Temporal evolution of intensity for different planes along the amplifier. The coherent $\mathrm{HH}$ beam is seeded at $10 \mathrm{ps}$ with $0.1 \mu \mathrm{J}$ energy and 200 ps duration. Most of the energy is clearly extracted by the coherent beam. 


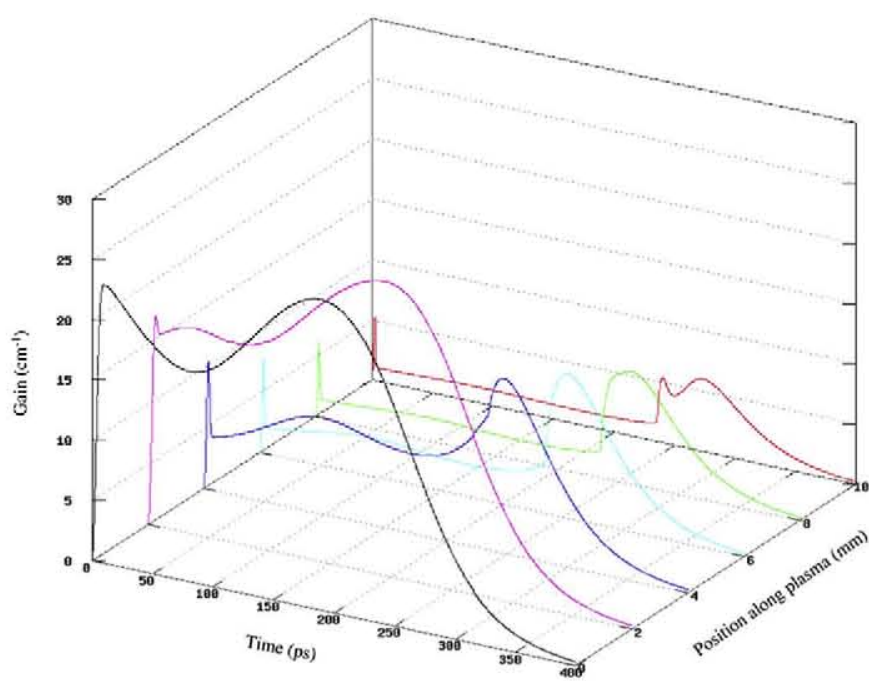

Fig. 6. Temporal evolution of the gain for different planes showing unambiguously that HHG seed saturates the gain instead of ASE for cases displayed in Figs. 2 and 4.

the spontaneous emission. We thus modelled this case considering the same plasma parameters as before and seed with $1 \mu \mathrm{J}$ of energy and 200 ps duration, which is the FWHM of the Gaussian pulse. The temporal evolution of intensity and gain versus position along the amplifier are displayed in Figs. 5 and 6. Gain curves show unambiguously that seed pulse extracts most of the stored energy by deeply saturating the gain. It is interesting to note that the gain saturates in a shorter distance than the previous case where a femtosecond seed was used, see Figs. 2 and 4 . At $6 \mathrm{~mm}$, the gain for femtosecond seed varies between 3 and $7 \mathrm{~cm}^{-1}$ while for long seed the gain remains below $2 \mathrm{~cm}^{-1}$. This may allow the use of shorter plasmas with the positive outcome of reducing the pump laser energy. To our knowledge the output energy of $5 \mathrm{~mJ}$ achieved with long seed is 4 orders of magnitude higher than the current record [8] reached by a fully coherent, in space and in time, soft $\mathrm{X}$-ray laser. This laser can be used as is (a $5 \mathrm{~mJ}$ coherent pulse of $\sim 100$ ps duration), replacing incoherent QSS soft x-ray laser still in operation that demonstrated great attractiveness to users despite poor coherence [6].

Finally, we would like to provide the first considerations for fielding this experiment. Seeding relies on the use of high harmonics generated by the interaction of femtosecond laser with rare gas. The HHG beam is both spatially and temporally coherent and may exhibit diffraction-limited wavefront [12]. However, in this configuration HHG typically exhibits a $20 \mathrm{fs}$ duration that is too short, as demonstrated here. The solution consists of setting up an architecture similar to Chirped Pulse Amplification, but here it is for short wavelengths. The HHG pulse duration can be increased by 100 times in a stretcher using a diffraction grating plus an imaging mirror, with conventional XUV optics [13]. Then the HHG are amplified and compressed to the Fourier-limit with a symmetric pair of diffraction gratings. In the modelled case, the Fourier-limit is about $130 \mathrm{fs}$ and considering classical gratings one may expect achieving about $3 \mathrm{~mJ}$ of energy.

\section{Conclusion}

In conclusion, we developed a time-dependent Bloch-Maxwell code to model the amplification of high harmonic seeding in a QSS zinc plasma that allows one to study both direct femtosecond and chirped configurations. We demonstrated that femtosecond seeding is inefficient for extracting energy in such amplifiers. CPA architecture applied to soft $x$-ray laser allows one to extract most of the stored energy $(6 \mathrm{~mJ})$ while keeping ASE at very low energy of about $10 \mu \mathrm{J}$. Compressed or not, the SXRL beam will have much better parameters, in terms of energy, coherence, polarization, than any current SXRL pumped by a laser.

\section{Acknowledgement}

The authors would like to thank Prof. G. Mourou and J.P. Chambarret for fruitful discussion on CPA technology. The work has been partially supported by the SFINX-LASERLAB European Community's Seventh Framework Programme under grant agreement $n^{\circ} 228334$.

\section{References}

[1] A.J. Nelson, et al., Soft x-ray free electron laser microfocus for exploring matter under extreme conditions, Optics Exp. 17 (20) (2009) 1827.

[2] L. Young, et al., Femtosecond electronic response of atoms to ultra-intense X-rays, Nature 466 (2010) 56.

[3] H.N. Chapman, et al., Femtosecond diffractive imaging with a soft-X-ray free electron laser, Nat. Phys. 2 (2006) 839.

[4] C. Bostedt, et al., Multistep ionization of argon clusters in intense femtosecond extreme ultraviolet pulses, Phys. Rev. Lett. 100 (2008) 133401.

[5] H. Daido, Review of soft x-ray laser researches and developments, Rep. Prog. Phys. 65 (2002) 1513-1576.

[6] B. Rus, et al., Multimillijoule, highly coherent $x$-ray laser at $21 \mathrm{~nm}$ operating in deep saturation through double-pass amplification, Phys. Rev. A 66 (2002) 063806.

[7] R. Benware, et al., Focusing of a tabletop soft-x-ray laser beam and laser ablation, Opt. Lett. 24 (23) (1999) 1714.

[8] P. Zeitoun, et al., A high-intensity highly coherent soft X-ray femtosecond laser seeded by a high harmonic beam, Nature 431 (2004) 426.

[9] Wang, et al., Phase-coherent, injection-seeded, table-top soft-X-ray lasers at $18.9 \mathrm{~nm}$ and $13.9 \mathrm{~nm}$, Nat. Photon. 2 (2008) 94.

[10] T. Ditmire, et al., Amplification of XUV harmonic radiation in a gallium amplifier, Phys. Rev. A 51 (1995) 6.

[11] D.S. Whittaker, et al., Reaching the $100 \mathrm{fs}$ frontier with intense, plasma-based soft X-ray lasers by seeding with ultra-short pulses, Phys. Rev. A 81 (2010) 043836.

[12] J.P. Goddet, et al., Aberration-free laser beam in the soft x-ray range, Opt. Lett. 32 (2007) 11.

[13] F. Frassetto, P. Villoresi, L. Poletto, Optical concept of a compressor for XUV pulses in the attosecond domain, Opt. Exp. 16 (9) (2008) 6652. 